\title{
RELATING THE MINIMAL ANNULUS WITH THE CIRCUMRADIUS OF A CONVEX SET
}

\section{A. Hernández Cifre and P. J. Herrero Piñeyro}

Abstract. In this paper we relate the minimal annulus of a planar convex body $K$ with its circumradius, obtaining all the upper and lower bounds, in terms of these quantities, for some of the classic geometric measures associated with the set: the diameter, the minimal width and the inradius. We prove the optimal inequalities for each one of those problems, determining also its corresponding extremal sets.

Mathematics subject classification (2010): 52A40, 52A10, 52A38.

Keywords and phrases: Convex bodies, minimal annulus, circumradius, diameter, minimal width, inradius, area, perimeter.

\section{REFERENCES}

[1] I. BÁRÁNY, On the minimal ring containing the boundary of a convex body, Acta Sci. Math. (Szeged), 52, 1-2 (1988), 93-100.

[2] T. Bonnesen, Les problèmes des isopérimètres et des isépiphanes, Collection de monographies sur la théorie des fonctions, Gauthier-Villars, Paris 1929.

[3] T. Bonnesen, W. Fenchel, Theorie der Konvexen Körper, Springer, Berlin 1934, 1974; Chelsea, New York 1948.

[4] J. FAVARD, Problèmes d'extremums relatifs aux courbes convexes I, II, Ann. École norm. 46 (1929), 345-369.

[5] J. FAVARD, Sur le déficit isopérimétrique maximum dans une couronne circulaire, Mat. Tidsskr. B (1929), 62-68.

[6] M. A. Hernández Cifre, P. Herrero, Some optimization problems for the minimal annulus of a convex set, Math. Ineq. Appl. 9 (2) (2006), 359-374.

[7] M. A. HERnÁndez Cifre, P. HERRERo, Optimizing geometric measures for fixed minimal annulus and inradius, Rev. Mat. Iberoamericana 23 (3) (2007), 953-971.

[8] N. KRitikos, Über konvexe Flächen und einschließende Kugeln, Math. Ann. 96 (1) (1927), $583-586$.

[9] C. PERI, On the minimal convex shell of a convex body, Canad. Math. Bull. 36 (4) (1993), 466-472.

[10] C. PERI, Minimal shells containing a convex surface in Minkowski space, Manuscripta Math. 90 (3) (1996), 333-342.

[11] C. Peri, S. VASSAllo, Minimal properties for convex annuli of plane convex curves, Arch. Math. (Basel) 64 (3) (1995), 254-263.

[12] C. PERI, A. ZuCCO, On the minimal convex annulus of a planar convex body, Monatsh. Math. 114 (2) (1992), 125-133.

[13] I. M. Yaglom, V. G. BoltyanskiĬ, Convex Figures, Holt, Rinehart and Winston, Russian original, Moscow-Leningrad 1951; New York 1961.

[14] A. ZuCCO, Minimal annulus of a convex body, Arch. Math. 52 (1) (1989), 92-94.

[15] A. Zucco, Minimal shell of a typical convex body, Proc. Amer. Math. Soc. 109 (3) (1990), $797-802$. 